\title{
Editorial and Comment Cost Containment and Patient Well-Being
}

\author{
Kevin R. Riggs, MD, MPH' ${ }^{1,2,3}$ and G. Caleb Alexander, $M D, M S^{1,2,4}$ \\ 'Division of General Internal Medicine, Johns Hopkins University School of Medicine, Baltimore, MD, USA; ${ }^{2}$ Center for Drug Safety and Effectiveness, \\ Johns Hopkins University, Baltimore, MD, USA; ${ }^{3}$ Johns Hopkins Berman Institute of Bioethics, Baltimore, MD, USA; ${ }^{4}$ Department of Epidemiology, Johns \\ Hopkins Bloomberg School of Public Health, Baltimore, MD, USA.
}

J Gen Intern Med 30(6):701-2

DOI: $10.1007 / \mathrm{s} 11606-015-3219-4$

(c) Society of General Internal Medicine 2015

$\mathrm{E}$ ver since policymakers began using health care reimbursement as a tool to contain health care costs, physicians have voiced concerns that the resulting financial incentives may conflict with their primary duty of advocating for their patients' wellbeing. ${ }^{1}$ At times, these concerns have had merit. For example, some of the less popular cost-containment mechanisms of the managed care era, such as aggressive gatekeeping, were abandoned in part because of their potential harm to patients. ${ }^{2}$

One cost-containment mechanism that has proven more resistant to physician concerns is the prospective payment of hospital care. Prospective payment was implemented in the Medicare program in 1983. By paying hospitals a flat fee based on the admission diagnosis, prospective payment reversed the financial incentives faced by hospitals: instead of being paid for each additional service provided to patients, hospitals could be more profitable when patients received fewer services and were discharged more quickly. After the average length of stay (LOS) for elderly patients fell from 10.7 days in 1980 to 8.7 days in $1985,{ }^{3}$ some questioned whether Medicare patients were being discharged "quicker and sicker."

However, within a decade of prospective payment implementation, published research had largely allayed the concern that it was harming patients. A series of studies comparing Medicare patients hospitalized with one of five diagnoses in 1981-1982 to a similar group hospitalized in 1985-1986 found that while there was a slight increase in patient instability at discharge, processes of care during hospitalization improved, and the absolute risk of 30-day mortality decreased by more than $1 \%{ }^{4}$ As a review of the early evidence summarized, "The literature offers some hope that the worst fears about the effects of [prospective payment] on quality of care... have not materialized."

In this issue of JGIM, Southern and Arnsten examine the association between inpatient length of stay and 30-day mortality, raising anew the concern that policies incentivizing short LOS may lead to worse patient outcomes. ${ }^{6}$ One everpresent concern in comparative analyses of outcomes between patients with short and long LOS is that these two groups of

Published online February 13, 2015 patients differ in unobserved and unobservable ways. In an effort to address this, the authors examined the outcomes of patients cared for by physicians with short LOS tendencies to patients cared for by physicians with long LOS tendencies. Using a retrospective cohort from their own institution comprising a total 12,341 admissions for ten common medical diagnoses treated by 79 physicians from 2002 to 2008 , the authors found increased risk of 30-day mortality in patients cared for by physicians with short LOS tendencies, with statistically significant increased odds of $33-43 \%$ in adjusted models, depending upon whether propensity score matching was used. The authors conclude that policymakers should weigh this increased risk when considering policies that incentivize shorter inpatient LOS.

To our knowledge, the grouped-treatment approach that the authors used has not previously been applied in this context, but their analytic design has several notable limitations nevertheless. First, while the intent was to capture a natural experiment (as admissions were essentially assigned to physicians at random), there were significant observable differences between patients cared for by the two groups of physicians, including patient sex, race, insurance status, number of comorbidities, and severity of illness at admission. Furthermore, there was an uncomfortably wide range in mean LOS among individual physicians (3.71 to 8.14 days!), suggesting that patients in the two groups may have differed in important unobserved ways. While the authors adjusted for patient and physician factors available to them, the results still may have been affected by residual confounding. Second, the authors derived the physician LOS tendencies from the final patient cohort. One alternative approach that might have diminished the potential for residual confounding would have been to determine LOS tendencies from an initial subset of patients and then compare outcomes in a separate cohort. Third, the authors attributed patients to the discharging physician, reasoning that they were the final arbiter of the decision to discharge. However, patients may have been treated by numerous attending physicians during their hospitalization, and it remains unclear whether this approach is optimal, both for determining a physician's LOS tendency and for attributing adverse events such as 30-day mortality. Additional sensitivity analyses, such as including only patients who received a majority of their treatment-days from a single physician, might have been useful given the novelty of this approach.

These limitations notwithstanding, it is also important to note that both groups of physicians in this study were 
operating within the same incentive environment. Thus, these findings should not be taken as evidence that incentives favoring shorter hospitalization accounted for any observed difference. In other words, even if relaxing the incentives to shorten LOS ultimately led to increased LOS, it is unclear whether this would improve outcomes for patients in either or both groups.

While unlikely to represent the death knell for prospective payment, this study offers important reminders for physicians, researchers, and policymakers. First, patient well-being should remain physicians' primary obligation. "Doing more" may not always be the best thing for patients, ${ }^{7}$ but when it is, physicians should not let financial consequences stop them from providing the appropriate care. After all, under the prospective payment system, hospitals are not intended to earn a profit from each admission; the system works because the profits from patients who require fewer than the average number of services more than offset the losses from patients who require more services.

Second, natural variation among practices creates opportunities for researchers to identify those practices that are most effective. ${ }^{8}$ Southern and Arnsten have reminded us that variation is everywhere, and once it is identified, delving deeper can offer insights into ways to improve health care. For example, there are several mechanisms that might account for worse outcomes for physicians with short LOS tendencies, assuming that threats to validity from residual confounding could be eliminated. Are those physicians discharging patients without adequate observation? Are they providing fewer beneficial therapies in the hospital? Or are worse outcomes a result of insufficient discharge planning and coordination? Determining best practices can help to improve the quality of care delivered by all physicians.

The era of cost containment is far from over, and the Affordable Care Act promises that the next decade will bring new models of care delivery and reimbursement. For example, accountable care organizations will be incentivized to achieve financial savings, but unlike managed care organizations of the 1990s, they will also be held to high standards on numerous quality metrics. ${ }^{9}$ Fortunately, as with managed care of the 1990s, new models of care are likely to be highly scrutinized, which will help ensure that patient well-being does not suffer in the name of cost containment.

Conflict of Interest: Dr. Alexander is Chair of the FDA's Peripheral and Central Nervous System Advisory Committee, serves as a paid consultant to IMS Health, and serves on an IMS Health scientific advisory board. This arrangement has been reviewed and approved by Johns Hopkins University in accordance with its conflict of interest policies.

Corresponding Author: Kevin R. Riggs, MD, MPH; Division of General Internal Medicine, Johns Hopkins University School of Medicine, 2024 E. Monument Street Room 2-604B, Baltimore, MD 21287, USA (e-mail: kriggs3@jhmi.edu).

\section{REFERENCES}

1. Alexander GC, Lantos JD. The doctor-patient relationship in the postmanaged care era. Am J Bioeth. 2006;6:29-32.

2. Young GP, Lowe RA. Adverse Outcomes of Managed Care Gatekeeping. Academic Emergency Medicine. 1997;4:1129-36.

3. Buie VC, Owings MF, DeFrances CJ, Golosinskiy A. National hospital discharge survey: 2006 annual summary. Vital Health Stat. 2010;13:1-79.

4. Rogers WH, Draper D, Kahn KL, et al. Quality of care before and after implementation of the DRG-based prospective payment system: a summary of effects. JAMA. 1990;264:1989-94.

5. Coulam RF, Gaumer GL. Medicare's prospective payment system: A critical appraisal. Health Care Financ Rev. 1992;1991(Suppl):45-77.

6. Southern WN, Arnsten JH. Increased Risk of Mortality among Patients Cared for by Physicians with Short Length-of-Stay Tendencies. Journal of General Internal Medicine 2015. [SPI 3155]

7. Fisher ES, Welch HG. Avoiding the unintended consequences of growth in medical care: how might more be worse? JAMA. 1999;281:446-53.

8. Tomson CR, van der Veer SN. Learning from practice variation to improve the quality of care. Clin Med. 2013;13:19-23.

9. Emanuel EJ. Why accountable care organizations are not 1990s managed care redux. JAMA. 2012;307:2263-4. 